\title{
Energy Efficient Radio Tomographic Imaging
}

\author{
Mojgan Khaledi, Sneha Kumar Kasera \\ School of Computing \\ University of Utah \\ Email: \{mojgankh, kasera\}@cs.utah.edu
}

\author{
Neal Patwari, Maurizio Bocca \\ Electrical and Computer Engineering Department \\ University of Utah \\ Email: \{npatwari, maurizio.bocca\}@ece.utah.edu
}

\begin{abstract}
In this paper, our goal is to develop approaches to reduce the energy consumption in Radio Tomographic Imaging (RTI)-based methods for device free localization without giving up localization accuracy. Our key idea is to only measure those links that are near the current location of the moving object being tracked. We propose two approaches to find the most effective links near the tracked object. In our first approach, we only consider links that are in an ellipse around the current velocity vector of the moving object. In our second approach, we only consider links that cross through a circle with radius $r$ from the current position of the moving object. Thus, rather than creating an attenuation image of the whole area in RTI, we only create the attenuation image for effective links in a small area close to the current location of the moving object. We also develop an adaptive algorithm for determining $r$. We evaluate the proposed approaches in terms of energy consumption and localization error in three different test areas. Our experimental results show that using our approach, we are able to save $50 \%$ to $80 \%$ of energy. Interestingly, we find that our radius-based approach actually increases the accuracy of localization.
\end{abstract}

\section{INTRODUCTION}

Radio frequency (RF) sensor networks can track people and objects without requiring them to carry any wireless transmit or receive devices [1]. Moving people and objects can be located based on the changes they cause in the received signal strength (RSS) of the radio links they perturb between transmit and receive sensor nodes. This "device free" localization of physical objects is called radio tomographic imaging (RTI) [2], [3], [4], [5], [6], [7], [8]. Localization of moving people and objects using RF sensor networks has several applications including surveillance, rescue operations, and residential monitoring [9].

One critical issue which has been neglected in existing RTI approaches is energy efficiency. RTI has primarily focused on location accuracy and assumed that sensors are connected to wall power sockets or their batteries can be recharged often. When deploying RTI in outdoor settings where wall power is not an option, saving energy of sensor nodes becomes a key requirement. It might not be easy to recharge sensor nodes after deploying them. Even in indoor environments, wall power might not always be available. Therefore, it is desirable to reduce the energy consumption of RTI sensor nodes as much as possible to prolong the lifetime of the RF sensor network. In this paper, our goal is to develop approaches to reduce the energy consumption in RTI methods without giving up accuracy.

A typical RTI set up deploys a mesh of $n$ transceivers around the area that is to be monitored. In this set up, each node takes turn to transmit radio signals. All the other

978-1-4799-4657-0/14/\$31.00 (c) 2014 IEEE nodes, when not transmitting, receive radio signals. The measurements of the RSS on all the $O\left(n^{2}\right)$ links between the transmitters and the receivers are used for tracking moving objects in the monitored area. We develop energy efficient target tracking methods that essentially limit the number of radio links that we must measure at any given time thereby allowing us to deactivate a large number of transceivers and hence save energy on these nodes. Our key idea is to only measure those links that are near the current location of the moving object. In order to find the effective links near to the current location of the moving object, we propose two approaches. In our first approach, we only consider links that are in an ellipse around the velocity vector from the current location of the moving object. In our second approach, we only consider links that cross through a circle with radius $r$ from the current position of the moving object. Thus, rather than creating an attenuation image of the whole area in RTI, we only create the attenuation image for effective links in a small area close to the current location of the moving object.

We propose an adaptive algorithm to change the value of $r$ in the radius-based approach. The value of $r$ must change over time depending on the velocity of the moving object, the number of links that cross through the circle, and the amount of error in the current location estimation. Moreover, the tracking accuracy and the energy consumption in the radius-based approach is highly depended on the value of $r$. For example, if we choose a large value for $r$ that includes all changes, then the accuracy of localization improves at the expense of relatively high energy consumption.

Our contributions in this paper are as follows. First, we define two energy efficient approaches for localization using RTI. Our energy efficient approaches can be used with both Shadowing-based RTI and variance-based RTI [2], [3], [4], [5], [6], [7], [8]. Second, we introduce an adaptive algorithm to change the radius in the radius-based approach. Third, we evaluate the proposed approaches in terms of energy consumption and localization error in three different test areas - an open indoor area, a cluttered office, and the aisles of the University of Utah campus bookstore. Our experimental results show that using our approach, we are able to save $50 \%$ to $80 \%$ of energy. Interestingly, we find that our radius-based approach for energy efficiency actually increases the accuracy of localization.

The rest of this paper is organized as follows. Section II contains the relevant related work. In Section III, we briefly describe two basic approaches (Shadowing-based RTI, and variance-based RTI) for localization using radio tomographic imaging. Section IV represents the energy efficient approaches 
in details. In Section V, we describe three conducted experiments and evaluate the results. Section VI is devoted to some directions for future work. Finally, the concluding remarks is provided in Section VII.

\section{RELATED WORK}

RF sensor networks estimate the location of people and objects using the changes in RSS measured on the links of a wireless network. A growing body of research has developed approaches to improve the robustness of RF sensing to the challenges of the multipath radio channel. Experimental systems have demonstrated locating a person in offices or homes with average errors from $17 \mathrm{~cm}$ to $1 \mathrm{~m} \mathrm{[10],} \mathrm{[11],} \mathrm{[5],} \mathrm{[12],} \mathrm{[6],}$ even across an entire office building floor [13], while tracking multiple people [14], [15], [16], [17], and even through exterior walls [7], [18], [19]. Moreover, measurements from standard wireless devices have been shown experimentally to enable reliable gesture recognition [20], breathing rate estimation [21], and fall detection [22], [23]. These experimental results are impressive despite the fact they are predominantly tested in the $2.4 \mathrm{GHz}$ band where the REM system experiences interference from WiFi.

Several general approaches exist for using RSS in an RF sensor network for localization. One is radio tomographic imaging (RTI), which estimates a map of the activity in the area of deployment, computed from the changes in mean [2], [3], [4], [5], [6] or variance [7], [8] of RSS. Another perspective is to use machine learning to estimate position, using a pre-recorded set of training data with a person in each position [13], [16], [23]. Another approach is to use line crossing information directly to infer position, either using a geometrical model [10], [15] or a statistical model for RSS given person location (and statistical inversion) [11], [12].

The energy consumption of RF sensor networks has been addressed by very few works, to our knowledge. Sensor nodes in RF networks have limited supply of energy and it is often difficult to recharge them after deployment. Thus, in order to increase the lifetime of network, the energy consumption should be reduced. One way to reduce the energy required for data collection is to have sensors locally process RSS data and decide when a link is crossed, and forward data only when a link is detected as crossed [24]. This idea is complementary to this proposed work, which would turn off sensors' RSS data collection if the link is not expected to be crossed due to current human positions. Compressed sensing (CS), which estimates tomographic images using fewer link measurements, has been tested for tomographic building structure imaging [25], [26] and for open-area attenuation-based RTI [27]. These papers explore sensing strategies that are fixed, even if random, not a function of the current positions of people. In contrast, we present an adaptive strategy. Future work might combine the benefits of both CS and adaptive strategies.

In contrast to energy efficiency in $R F$ sensor networks, research in energy efficiency in wireless sensor networks (WSN) is significantly more mature [28], [29], [30]. A general WSN uses transmission and reception purely for data communication, not to measure the environment as in an RF sensor network. In an RF sensor network, it is insufficient to find a low-energy route from source to destination. Instead, we have a tradeoff between activating transmitter and receivers to minimize tracking error, and allowing them to sleep to minimize energy consumption. The adaptive algorithm explored in this paper addresses this fundamental tradeoff.

\section{BACKGROUND ON RADIO TOMOGRAPHIC IMAGING}

Radio tomographic imaging is the process of imaging the attenuation caused by physical objects moving in an RF sensor network. This image can be used to find the location of the moving objects or people within the area of deployed RF network.

In an RF network with $n$ static sensor nodes, there are $L=$ $n(n-1)$ directed links. Let vector $y=\left[y_{1}, y_{2}, \ldots, y_{L}\right]^{T}$ be the measurement for all links. Also, let $x=\left[x_{1}, x_{2}, \ldots, x_{M}\right]^{T}$ be the vector of voxels values, where $M$ is number of voxels. Then, $y$ can be modeled as:

$$
y=W x+n
$$

Where, $n$ is $L \times 1$ vector that represents the noise level of $L$ links. $W$ is a $L \times M$ matrix where $w_{i j}$ indicates how the voxel $j$ 's attenuation affects link $i$.

The weight matrix, $W$, is modeled by an ellipse [3], [6], [7]. In this model, an ellipsoid with foci at the transmitter and receiver locations determines the weight for each link. If voxel $j$ falls outside of link $i$ 's ellipsoid, $w_{i j}$ is set to zero. Otherwise, $w_{i j}$ is set to the constant, which is inversely proportional to the square root of the link length, as follows:

$$
w_{i j}=\left\{\begin{array}{lc}
\frac{1}{\sqrt{d}} & d_{r j}+d_{t j}<d+\lambda \\
0 & \text { otherwise }
\end{array}\right.
$$

Here, $d$ is the length of link $i, d_{r j}$ and $d_{t j}$ are length of line from the center of voxel $j$ to the receiver and transmitter locations of link $i$, respectively, and $\lambda$ represents the width of the ellipse.

In the shadowing-based RTI, $y$ is the change in RSS mean values and in variance-based RTI, $y$ is the windowed variance of RSS on each link. The image vector $x$ is estimated from $y$. However, finding $y$ is an ill-posed inverse problem. Thus, regularization is required to reduce the noise in the image. A regularized least square approach [2], [8] is used for both shadowing-based RTI and variance-based RTI:

$$
\begin{aligned}
\hat{x} & =\Pi y \\
\Pi & =\left(W^{T} W+\sigma_{N}^{2} C_{x}^{-1}\right)^{-1} W^{T}
\end{aligned}
$$

in which $\sigma_{N}^{2}$ is the noise variance. The prior covariance matrix $C_{x}$ is obtained by using an exponential spatial decay:

$$
\left[C_{x}\right]_{j l}=\sigma_{x}^{2} e^{-d_{j l} / \delta_{c}}
$$

where $\delta_{c}$ tunes the amount of smoothness in the image, $\sigma_{x}^{2}$ is the variance of voxel attenuation, and $d_{j l}$ is the length of line between voxel $j$ 's center and voxel $l$ 's center. 


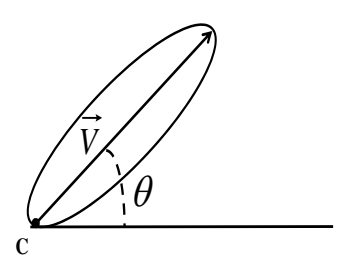

Fig. 1. An Ellipse around the velocity vector from the current location of the moving object

\section{ENERGY EFFICIENT RTI APPROACHES}

In this section, we describe two energy efficient approach for RTI that limit the number of radio links that we must measure at any give time thereby allowing to deactivate a large number of transceivers and hence save energy on those nodes. In the basic RTI approach, all transceivers are active at all times.

To reduce the energy consumption, instead of forming the image of attenuation for the whole monitored area, we only construct the image of attenuation for the small area near the current position of the moving object. We expect a temporal dependency in the moving pattern of object. Therefore, the next location of the moving object is likely to be close to its current location. Additionally, the moving object only changes the RSS of the links that are near the moving area. In fact, as the distance of a link from the moving object increases, it provides less information about the attenuation caused by the moving object. Thus, only links that are within a certain distance from the current location of the moving object contribute to the formation of the attenuation image. We refer to these links as effective links. Our aim is to save energy by measuring only the effective links and deactivate those transceivers that do not construe these links.

To find the effective links, we use two approaches. In the first approach, the effective links are those that cross through the ellipse around the velocity vector from the current location of the moving object. In the second approach, effective links are within the circle of radius $r$ from the current location. We explain these two approaches in Sections IV-A and IV-B, respectively. Then, in Section IV-C, we describe a scheduling policy for activating and deactivating appropriate transceivers to reduce the energy consumption.

\section{A. Ellipse-based Approach}

Using the fact that a typical moving object changes its velocity and its direction of movement smoothly and gently instead of abruptly, in the ellipse-based approach, we only consider an ellipse around the velocity vector from the current position of the moving object to form an attenuation image for localization. Figure 1 shows the ellipse where $\vec{v}$ and $\theta$ are the velocity vector and direction of movement, respectively. The point $c$ is the current location of the moving object.

To estimate the velocity vector, we use the history of movements over a window of time $t$. The velocity vector at time $t+1$ is a function of the moving object locations during $[1, t]$ as follows:

$$
\begin{aligned}
& \vec{v}_{t+1}=\vec{v}_{t}+\epsilon \\
& \epsilon \sim N\left(0, \sigma^{2}\right)
\end{aligned}
$$

$\vec{v}_{t}$ is obtained by dividing the path length by the time taken to traverse the path. A path is the longest straight line that the moving object can traverse without changing the direction or pausing. Since the object need necessarily not move in a straight line and there are likely to be some deflections around the straight line, we are likely to see some small paths around the straight line. To address these deflections, we fit a straight line between two points with distance greater than $l_{\text {min }}$ such that the other points between these two points are a distance less than $w$ from the line.

We first run the basic RTI approach for $t$ seconds. Using the location histories of the moving object over time $t$, we estimate the velocity vector at time $t+1$. After that, we only consider links that are in the ellipse around the velocity vector. Again, in time period $t+2$, we set the window for estimating the velocity vector by 1 . Given that we use the ellipse-based approach from time $t+1$ onwards, our estimation of the velocity vector can contain errors. This error can gradually increase and the ellipse that we use can move far away from the actual location of the moving object.

To tackle this potential for increase in error, we periodically (with time $T>t$ ) compare the result of localization of our approach with the result of localization of the basic RTI approach. If the difference between the positions obtained by the two approaches is greater than a threshold, we run the basic RTI approach for time $t$ to obtain a more accurate history of movement of the object. Although, this re-estimation of the history reduces the localization error, it also increases the energy consumption because we run the basic approach more often.

The ellipse approach can reduce the number of measured links significantly and consequently, it can save energy. However, it is expected to work well only in scenarios where the changes in the mobility pattern are not drastic. In cases with drastic movements, e.g., an object moving back and forth or an object changing its direction of movement significantly because of the obstacle in the area, the localization error of the ellipse approach can be high. In a high mobility environment, the ellipse approach can consume more energy because of the need to run the basic RTI approach every $T$ time units. Essentially, the ellipse-based approach can result in high localization errors or high energy use when the tracked object exhibits an unpredictable movement pattern. For robustness in these mobility scenarios, we propose the radius-based approach in the next section.

\section{B. Radius-based Approach}

In the radius-based approach the effective links are those that are in a circle with radius $r$ from the current position of the moving object. Using a circle instead of ellipse reduces the error of localization in case of high changes in the mobility pattern. In the radius based approach, we start with the basic RTI approach by measuring all links. However, after finding the current location of the moving object, we only consider links that are in the circle with radius $r$ from the current location.

One important challenge in the radius-based approach is the determination $r$. An improper value of $r$ can increases the energy consumption or the localization error substantially. 


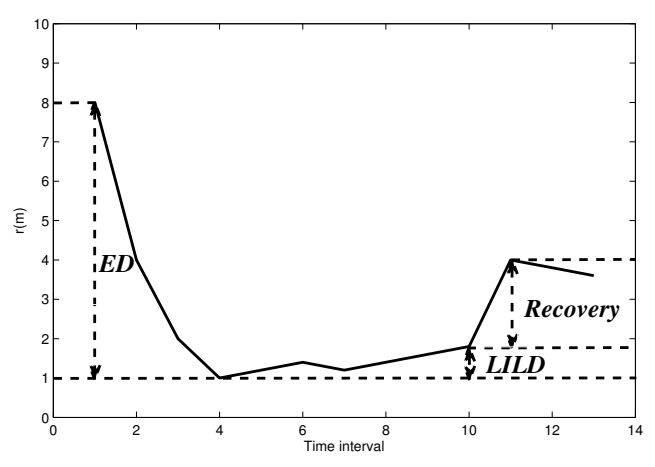

Fig. 2. The value of $r$ in each time period $T$

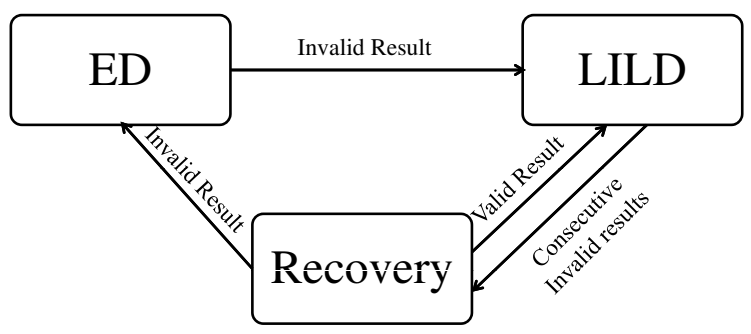

Fig. 3. Adaptive Algorithm for selection of $r$

Furthermore, the value of $r$ should be adapted dynamically depending on (i) the number of sensor nodes that are deployed, (ii) the obstacles (e.g., bookshelf, table, sofa) that are placed in the area, (iii) the number of links that cross the circle, (iv) the velocity of movement, and (v) amount of error in location estimation. Unlike the ellipse-based approach, we find the value of $r$ in the real time without using history of movement. Thus, the localization error does not increase over time. In the next section, we describe our adaptive algorithm for the selection of $r$.

1) Adaptive Algorithm for Radius Selection: In our adaptive algorithm for radius selection, we compare the location estimated using the radius-based approach with that estimated using the basic RTI approach every $T$ time units. We change the value of $r$ based on the difference between the location estimates and also based on the current state of the algorithm. Figure 3 shows the three states that we use in our adaptive algorithm to change the value of $r$. The algorithm starts in an exponential decrease, ED, state and then it goes to the linear increase linear decrease, LILD, state and then to the recovery state.

$\boldsymbol{E D}$ : The first state of algorithm is ED. In this state, we set the initial value of $r$ to $r_{\max } . r_{\max }$ is the radius of circle that covers the whole area of experiment and also all links, i.e, at the first step of ED both the radius-based and basic RTI approaches operate in a similar fashion. Then, in each time period $T$, we estimate the location using the radius-based approach and compare it with that obtained from the basic RTI approach. If the difference in the estimation is within a threshold, we reduce the value of $r$ by half, otherwise we go to the LILD state. Let $r_{k-1}$ denotes the value of $r$ after $k-1$ time units, the next value of $r$ in the ED state will be:

$$
r_{k}=\max \left(\alpha * r_{k-1}, r_{\text {min }}\right)
$$

Here, $\alpha=0.5$ is the exponential factor and $r_{\min }$ is the minimum value of $r$.

The ED state finds the range of $r$ which depends on several factors. We start with $r=r_{\max }$ to cover the whole area. However, due to temporal dependency and the fact that the moving object only changes the RSS of neighboring links, $r_{\max }$ is much greater that the optimal value for $r$. Thus, in each time interval we exponentially reduce the current value of $r$ when the difference in location estimates (in comparison to basic RTI) is low. When we go to the LILD step after $k$ time periods, the range of $r$ is determined to be from $r_{k-1}$ to $r_{k}$.

LILD: Given that we determine the range of $r,\left[r_{k-1}, r_{k}\right]$, quickly through exponential decrease in the ED state, we now adjust the value of $r$ much more slowly, by increasing or decreasing its values linearly, to move closer to its optimal value. The value of $r$ in this state is obtained as follows:

$$
r_{k}= \begin{cases}\max \left(r_{k-1}+\beta, r_{\text {min }}\right) & \text { Invalid Result } \\ \max \left(r_{k-1}-\beta, r_{\text {min }}\right) & \text { Valid Result }\end{cases}
$$

An invalid result in the above equation refers to the situation when the difference in location estimation using the radius-based and basic RTI approaches is greater than a threshold. A valid result is one where the difference in location estimation is less than or equal to the threshold. Every $T$ time units, we increase the value of $r$ linearly if the result is invalid and decrease the value of $r$ linearly if the result is valid. Thus, in LILD, the value of $r$ fluctuates between $r_{k-1}$ and $r_{k}$.

In the LILD state, it is possible that we observe consecutive invalid results. If the number of consecutive invalid results, despite linearly increasing $r$, is greater than a predefined threshold then we conclude that we are not converging to the right $r$. This means that the current location of the moving object is far from its true location and even by increasing the value of $r$ linearly in consecutive time periods, we are unable to find the location of the moving object correctly. In such a situation, stay in the LILD state will dramatically decrease the accuracy of localization. To avoid this, when we receive a certain number of consecutive invalid results, we go to the ED state again and set $r$ to $r_{\max }$. In other words, we start with the basic RTI approach to find the current location and reduce the value of $r$ exponentially to find its right range all over again.

Recovery: Although, in the ED state, we decrease the value of $r$ exponentially, it takes a long time for ED state to find the proper value for $r$ and consequently the sensor nodes consumes more energy. Thus, we should reduce the number of times that we go to the ED state. For this, we add the recovery state in the return path from the LILD to the ED state. Recovery state gives us a less drastic approach to find the proper value of $r$ before going to the ED state.

In this state, we double the current value of $r$ and then compare the result of localization after one time period $T$. If the result is valid then we go to the LILD state, otherwise we go to the ED state.

Figure 2 shows the changes of the value of $r$ in these three states over time. We first start with the ED state by setting the $r$ to $r_{\max }=8 \mathrm{~m}$. Then, we reduce the value of $r$ to half in each time slot. In this figure after four time units, the algorithm 

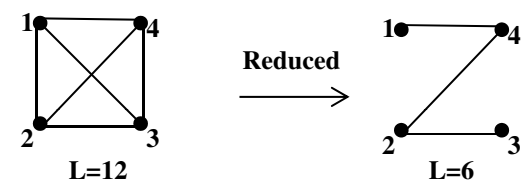

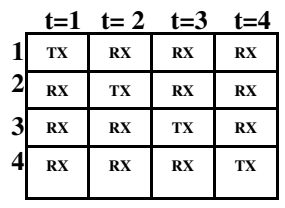
approach
One round of basic

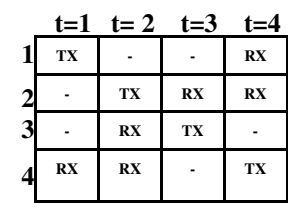

One round of energy efficient approach
Fig. 4. The scheduling policy for basic approach and energy efficient approach in an RF network with 4 sensor nodes

is able to find the range of changes for $r$. In the LILD state, we change the value of $r$ linearly. Finally, we double the value of $r$ in the recovery state.

\section{Scheduling}

In this section, we explain our scheduling policy for activating and deactivating the RF transceivers in our energy efficient approaches.

In the basic RTI approach, all sensor nodes are on at all times whether they transmit or receive signals. Figure 4 shows the scheduling policy for the basic RTI approach. In this figure, the deployed RF sensor network has 4 sensor nodes. To estimate the image of attenuation in the basic RTI approach, we must measure the RSS on all links in a full mesh topology. Considering the fact that in RF sensor networks the RSS of the link from $a$ to $b$ can be different from the RSS of the link from $b$ to $a$, in the basic RTI approach we must measure $L=12$ links.

Our scheduling policy for activating or deactivating sensor nodes is based on the Spin protocol [31]. The Spin protocol uses a token-based approach to prevent multiple sensor nodes from transmitting at the same time. In this protocol, the sensor nodes transmit in time division multiple access (TDMA) fashion with an order identified by their node IDs. When one sensor node is transmitting, all the other nodes are in the receiving mode. Figure 4 shows one round of scheduling in the basic RTI approach. In the first time slot, $t=1$, the first node is in transmitting mode (TX), and all the other nodes $(2,3$, and 4$)$ are in the receiving mode (RX). At the end of one round, in case multiple channels are used, the sensor nodes switch synchronously to the next frequency channel defined by the user and use the same scheduling as the previous channel. As shown in figure 4 , in all time slots, all nodes are either in the transmitting or in the receiving mode.

Our energy efficient approaches are based on the fact that not all links in the full mesh topology are effective. Independent of how we find the effective links (using the ellipse-based or the radius-based approach), Figure 4 shows one round of the scheduling policy for the graph with reduced links $(L=6)$. In the first time slot, $t=1$, node 1 is in the transmitting mode, node 2 and 3 are in the sleeping mode (deactivated), and node 4 is in the receiving mode. As this figure shows, in one round of an energy efficient approach, we can save $50 \%$ energy by increasing the number of times that sensor nodes are in the sleeping mode from 0 to 6 .

As shown in Figure 4, in energy efficient approaches, reducing the number measured links does not change the number of nodes that are in transmitting mode. This happens because in most real cases, sensor nodes are placed uniformly along the perimeter. For scheduling the receivers, at the beginning of each round, the sink node sends a small control packet to all nodes. This packet determines the receiving nodes in each time slot. Let $n$ be the number of sensor nodes, then size of the control packet for each node is $n$ bits. A bit is set to 1 , if the sensor node is in the receiving mode in that time slot, otherwise, it is set to 0 . Note the control packet is the same for all available channels and the sink node only sends it once for all channels, in case multiple channels are used for RTI.

If the sensor nodes are placed non-uniformly, then there might be some cases where the number of nodes that are in transmitting and receiving modes is reduced. In this case, the scheduling policy is entirely determined by the control packet that the sink node sends to all nodes. The control packet in this case indicates three modes (transmitting, receiving, sleeping) for each node in each time slot. Thus, the size of control packet for each node is $2 n$ bits in a network with $n$ nodes as 2-bits are required to represent three modes.

Note that the energy efficient approaches determine which bits should be set in the control packet based on the RSS of the measured links and the steps of these approaches. Once the control packet is created, it is sent to the sink node for distribution to the sensor nodes. The time complexity of the energy efficient approach is no worse than the basic RTI approach that is used for real time localization. In fact, the time complexity of the energy efficient approach is better than the basic approach. This is because, in most cases, the actual monitored area in the energy efficient approach is much smaller than the monitored area in the basic RTI approach.

\section{EVALUATION}

To evaluate the energy efficient approaches, we conduct experiments in three different areas: an open environment, a cluttered office area, and a bookstore. In this section, we first describe these three areas and then present the evaluations of our energy efficient approaches in terms of energy consumption and localization error in these three areas.

\section{A. Experiment areas}

Open Environment: In the open environment, there are no objects or obstructions in the monitoring area. Figure 5(a) shows the layout of this experiment. As shown in this figure, 30 sensor nodes are deployed along the perimeter of a $70 \mathrm{~m}^{2}$ area at the height of one meter from the floor. The sensor nodes transmits on channels $11,15,18,22$ and 26. The markers in Figure 5(a) shows the true positions of the moving person. The person starts moving at point $A$, then moves along the straight lines from $A$ to $B, B$ to $C, C$ to $D$, and $D$ to $A$. Finally, the person stops moving at point $A$. At each location, the person stands for $20 \mathrm{~s}$. We also measure the RSS of all links when no 


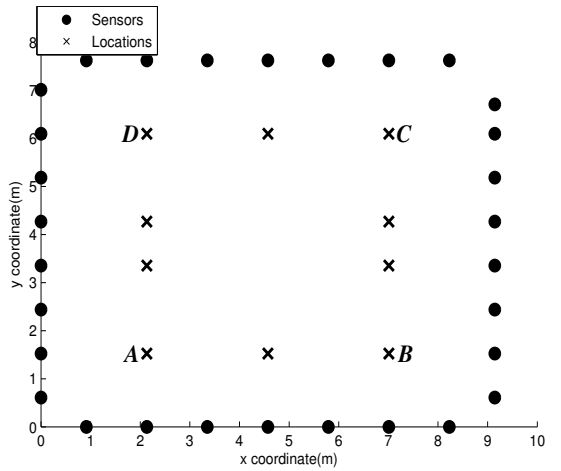

(a) Open Environment

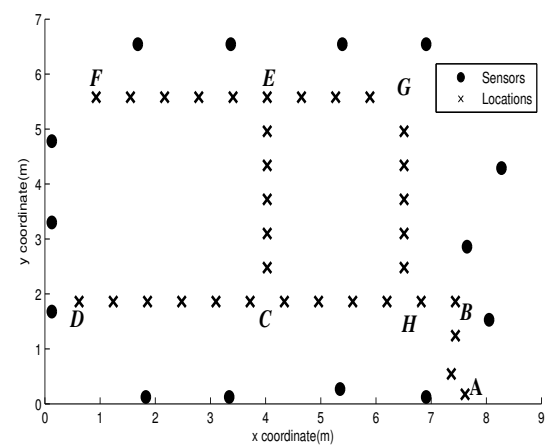

(b) Cluttered Office

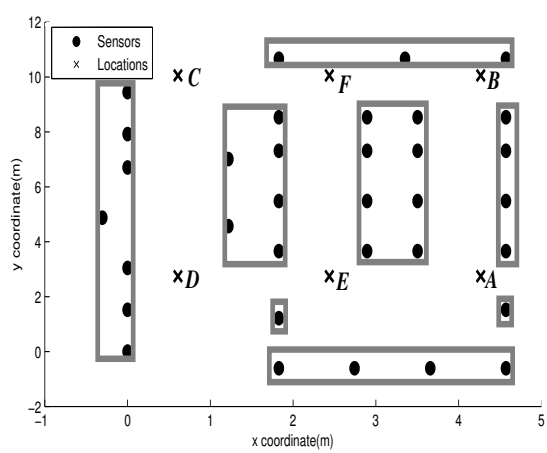

(c) Bookstore

Fig. 5. The layout and sensor nodes positions for open environment, cluttered office, and bookstore

person is present in the monitoring area for $60 \mathrm{~s}$ and use it for RTI calibration.

Cluttered Office: This experiment is done in a cluttered area where there are several metallic obstructions such as desks, chairs, and monitors. In this experiment, 14 sensor nodes are deployed inside of a $52 \mathrm{~m}^{2}$ area at the height of one meter from the floor, as shown in Figure 5(b). The sensor nodes transmit on channels $11,16,21$, and 26 . The marker points in Figure $5(b)$ shows the true locations. The person moves along the path $A B D C E F G H C E G B A$. As it can be seen in the movement path, in this experimental set up, the person changes the direction of movement more often than in the open environment. Using these two experiments, we are able to compare the results of ellipse and radius-based approaches in conditions where there are too many changes in the movement pattern (cluttered office) with the case where the changes in the movement pattern are minimal (open environment).

Bookstore: This experiment is performed in the University of Utah Bookstore in a $55 \mathrm{~m}^{2}$ area. As in the case of the office environment, the bookstore is cluttered with shelves, tables, and books. There are 34 sensor nodes that are positioned in the area, as shown in Figure 5(c). The gray rectangles in this figure are shelves. The sensor nodes transmit only on one channel. In one experiment (that we call Exp. 1), a person moves along the path $A B C D A$ twice and in the another experiment (that we call Exp. 2), the person moves along the path EFBAE twice.

In the first two experiments (open environment, and cluttered office), the sensors are TI CC2531 USB dongle nodes [32] and in the third experiment, bookstore, the sensor nodes are TelosB [33].

\section{B. Experimental results}

In this section, we evaluate the energy efficient approaches in terms of energy consumption and the localization error. For evaluation, we compare our work with both Shadowing-based RTI and variance based RTI approaches. We use the approach that is proposed in [6] from the shadowing based approaches and VRTI [7] from the variance based RTI approaches. In [6], the authors use channel diversity to improve the accuracy of localization in RTI.
TABLE I. PARAMETERS USED IN THE EXPERIMENTS

\begin{tabular}{ccccc}
\hline \hline Parameter & Description & Open & Office & Bookstore \\
\hline$P$ & Pixel width(m) & 0.15 & 0.5 & 0.5 \\
$\lambda$ & $\begin{array}{c}\text { Excess path length limit } \\
\text { of ellipse weighting(m) }\end{array}$ & 0.02 & 0.02 & 0.02 \\
$\sigma_{x}$ & Voxels variance(dB) & 0.05 & 0.1 & 0.1 \\
$\sigma_{N}^{2}$ & Noise variance(dB) & 1 & 1 & 1 \\
$\delta_{c}$ & Correlation coefficient & 4 & 4 & 4 \\
$m$ & Number of used channels & 4 & 4 & 1 \\
$\alpha$ & Exponential factor & 0.5 & 0.5 & 0.5 \\
$\beta$ & Linear factor(m) & 0.5 & 0.5 & 0.5 \\
$T$ & Time periods for RTI & 50 & 50 & 50 \\
$r_{m} i n$ & Min radius(m) & 0.5 & 0.5 & 0.5 \\
$t$ & Window size for ellipse & 5 & 5 & 5 \\
\hline
\end{tabular}

In order to evaluate the energy efficient approach, we use two metrics: average error of location estimation, and energy consumption ratio. The energy consumption ratio is obtained by dividing the total energy consumption in the energy efficient approach by the total energy consumption in the basic approach (e.g., Shadowing-based RTI, and variancebased RTI). The total energy consumption in an RF network with $n$ nodes is obtained from the following formula.

$$
E=\sum_{t=1}^{T} \sum_{i=1}^{n} E_{T x}(i, t)+E_{R x}(i, t)
$$

Here, $E_{T x}$ and $E_{R x}$ are energy consumptions in the transmitting and receiving modes respectively. We set both $E_{T x}$ and $E_{R x}$ to $4.5 \mathrm{~J}$ in all experiments. Table I shows the value of parameters used in these three experiments.

Figure 6(a) shows the energy consumption of the ellipse, radius-based, and the multi-channel RTI approaches in the open environment. This figure shows that both ellipse and radius based approaches can reduce the energy consumption ratio from 1 to 0.2 . In other words, both energy efficient approaches save $80 \%$ of energy compared to the multi-channel RTI approach. Also, this figure shows that there is not too much difference between the energy consumption ratio of ellipse and radius-based approach (see the yellow area). 


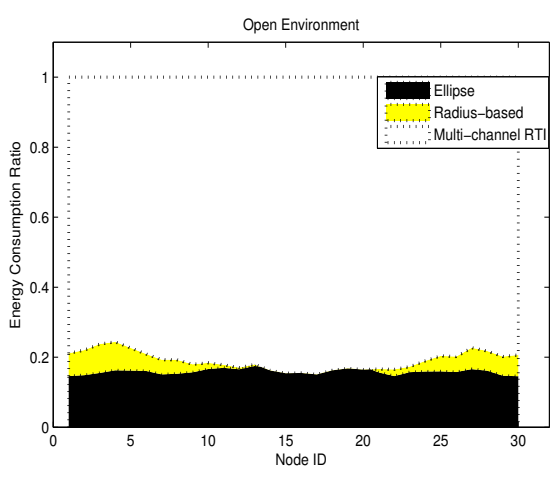

(a) Open Environment

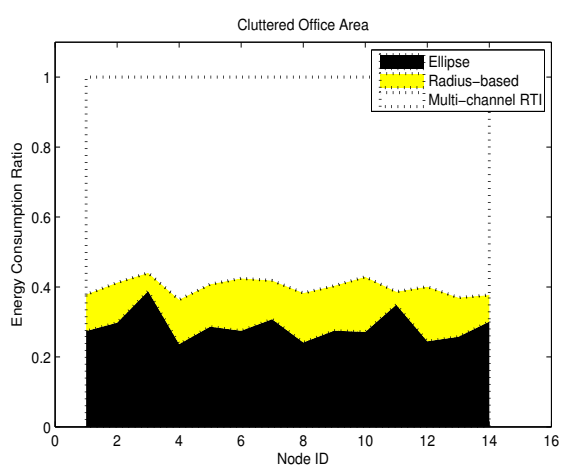

(b) Cluttered Office

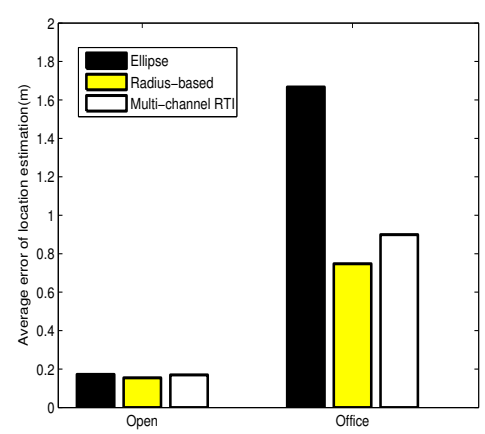

(c) Average error of location estimation

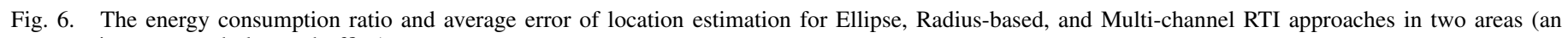
open environment and cluttered office)

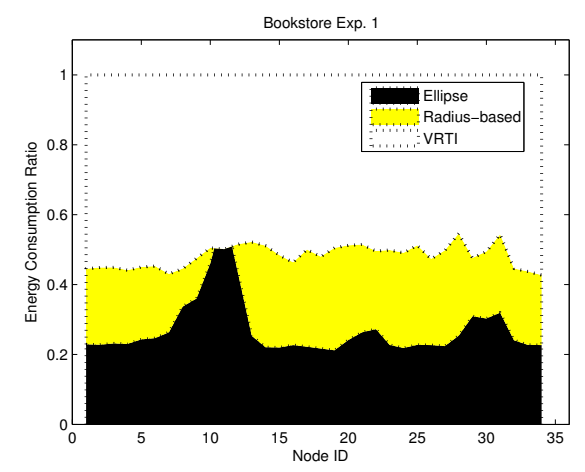

(a) Exp.1

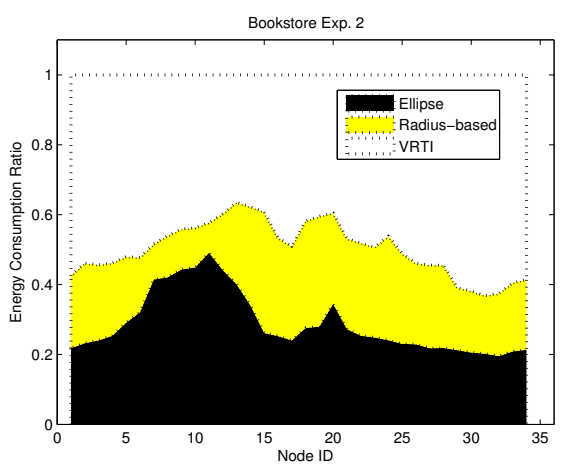

(b) Exp.2

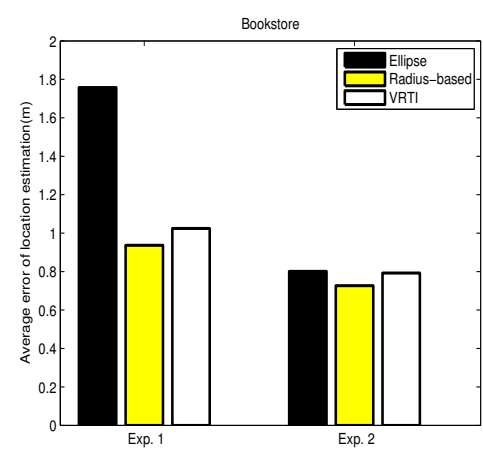

(c) Average error of location estimation

Fig. 7. The energy consumption ratio and average error of location estimation for Ellipse, Radius-based, and VRTI approaches in bookstore

Figure 6(b), compares the energy consumption ratio of the three approaches (the ellipse, radius-based, and multi-channel RTI) in a cluttered office area. This figure shows that the energy efficient approaches save around $60 \%$ of energy which is a little less than the energy savings in the open environment. This is because in the office area there are only 14 nodes which is significantly less than the number of nodes in the open area. The reduction in the number of measured links in the office area is less than that in the open area and therefore, we save less energy in the office area.

Figure 6(c) shows the average error of location estimation in the ellipse, radius-based and multi-channel RTI approaches for both open and cluttered office areas. In both areas (open environment and office), the error of radius based approach is slightly less than the multi-channel RTI approach. Since in the radius-based approach we only construct the image of attenuation for a circle around the current position, the noise in the other places does not effect the result of localization. However, in multi-channel RTI, we consider the whole area for constructing the image of attenuation and sometimes the noise in the environment corrupts the image and increases the error of localization. In addition, as shown in Figure 6(c), the average error of localization in the ellipse approach is slightly higher than the average error in multi-channel RTI approach for the open environment and it is a lot higher than the error of multi-channel RTI approach in the office environment. As we discussed earlier, one drawback of the ellipse approach is propagation of error. This propagation has high impact when we have frequent significant changes in the movement pattern. In the office area, the person changes the direction of movement more frequently compared to the open environment. Thus, the error of localization increases significantly in the office area.

Figures 7(a), 7(b), and 7(c) show the comparison of ellipse, radius-based, and VRTI approaches in the bookstore. Figures 7(a) and 7(b) show that by using the VRTI approach with ellipse and radius-based approach, we can save up to $50 \%$ of energy on average compared to the basic VRTI approach. However, the amount of reduction in the energy consumption is less than that in the open environment where we use multichannel RTI with the energy efficient approaches. This happens because in the VRTI approach, we need the RSS of links for a couple of rounds to be able to compute the variance. Therefore, in the energy efficient approaches over each $T$ time units, when we compare the basic approach with the energy efficient approach, we need to turn on all sensor nodes for a couple of rounds. This increases the energy consumption in this scenario. However, we still cut down the energy usage by half.

As shown in Figures 7(a) and 7(b), sensor nodes in Exp. 2 consume more energy than in Exp. 1. As mentioned in the previous section, in Exp. 1, the person moves along the path 
TABLE II. COMPARISON OF DIFFERENT APPROACHES

\begin{tabular}{c|cc|cc|cc|cc}
\hline \hline & \multicolumn{2}{|c|}{ Open } & \multicolumn{2}{c|}{ Office } & \multicolumn{2}{c|}{ Bookstore Exp.1 } & \multicolumn{2}{c}{ Bookstore Exp.2 } \\
\hline Approaches & $\bar{e}_{l o c}(m)$ & $\bar{E}_{R}$ & $\bar{e}_{l o c}(m)$ & $\bar{E}_{R}$ & $\bar{e}_{l o c}(m)$ & $\bar{E}_{R}$ & $\bar{e}_{l o c}(m)$ & $\bar{E}_{R}$ \\
\hline Ellipse & 0.172 & 0.1611 & 1.667 & 0.2862 & 1.7571 & 0.2715 & 0.8013 & 0.2871 \\
\hline Radius-based & 0.1544 & 0.1899 & 0.7481 & 0.3998 & 0.9368 & 0.4823 & 0.7264 & 0.5029 \\
\hline Basic Approach & 0.1693 & 1 & 0.8993 & 1 & 1.0245 & 1 & 0.7928 & 1 \\
\hline
\end{tabular}

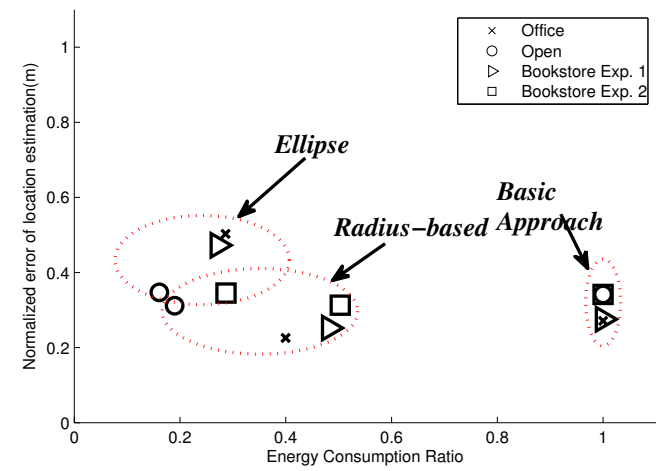

Fig. 8. Comparison between Ellipse, Radius-based, and basic approaches in terms of the total energy consumption ratio and localization error

$A B C D A$ that covers the whole area. However, in Exp. 2, the person moves along the path $E F B A E$ that is only a part of the whole area that is surrounded by lots of sensor nodes. Therefore, the reduction in the number of measured links in Exp. 2 is less than the reduction in Exp. 1. As a result, in Exp. 2, we consume more energy compared to that in Exp. 1. Also, in the bookstore experiment there is a higher difference in the energy consumption ratio of nodes. This happens because in the bookstore environment the sensor nodes are placed nonuniformly.

Table II shows the average location estimation error, $\bar{e}_{l o c}$ and the total energy consumption ratio, $\bar{E}_{R}$, in all the experiments. If we only consider the energy consumption ratio, the ellipse approach is better that the other approaches. However, the ellipse approach does not preform well in terms of localization. As we can see in this table, in all experiments the localization error in the ellipse approach is higher than the basic and the radius-based approaches and in some cases such as the office area the localization error is significantly higher than the error in the other approaches. In terms of localization, the radius-based approach performs better than the others. Also, it can save $50 \%$ energy in the worst case which is great. Figure 8 shows this comparison. In this figure, the $x$ axis represents the total energy consumption ratio, and the $y$ axis represents the normalized localization error. Our goal is to find an approach with points on the left bottom corner of the figure. This figure shows that the basic approach is on the right and close to the bottom. Our energy efficient approaches when applied to the basic approach, moves the points from the right to the left. Also, we decrease the distance from the bottom in the radius based approach.

\section{FUTURE WORK}

Several future research directions exist for energy efficient target tracking using RF sensor networks. First, in our work we proposed energy efficient approaches for tracking a single target only. However, in many real-world environments and

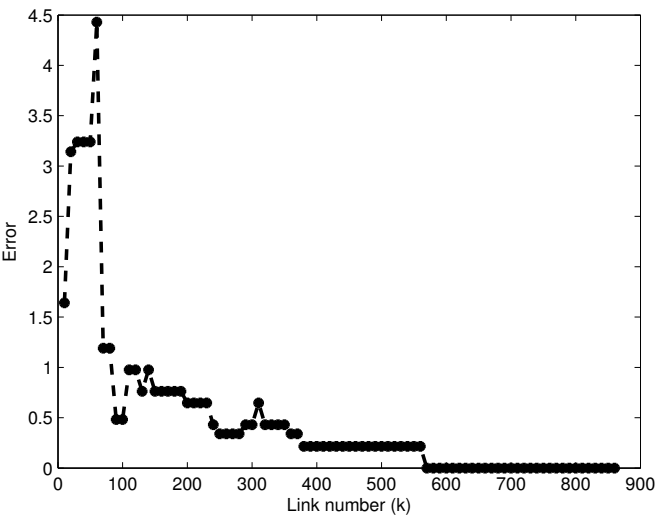

Fig. 9. The difference between the localization results when we use all links versus when we use the $k$ shortest links

settings, multiple people or objects are expected to move and be tracked in the monitored areas. While there is growing work on multiple target tracking [14], [17], energy efficiency in these scenarios has not received much attention. In the future, we want to extend our energy efficient approach to the multiple target tracking.

Second, we want to improve our energy efficient approach in terms of both the energy consumption and the error of localization. Currently, we reduce the energy consumption to half in the worst case. One way to reduce both the energy consumption and the localization error is by reducing the number of measured links even within the ellipse or the circle. Our idea is to assign weights to individual links and select a subset of them based on their weights. For example, we can weigh links based on their lengths. Short length links provide more information about the attenuation compared to the long ones. Figure 9 shows the difference in the localization error when we use all links that are crossing the monitored area versus the cases when we use $k$ links in the monitored area. The $k$ links are selected using shortest links first. In Figure 9, the total number of links is 870 . This figure shows that increasing the number of links from 570 to 870 does not change the estimated position. However, not all short links are informative (e.g., the short links that cross metallic obstructions can actually negatively impact the accuracy). Thus, the location of the link or its fade level is very important. Therefore, we can weigh links based on multiple metrics, use the most effective links, and thereby save more in terms of energy without sacrificing accuracy.

Third, besides reducing the total energy consumption, we want to distribute this energy benefit uniformly across the sensor nodes. In our current energy efficient approaches, the maximum energy consumption among the sensor nodes is half of that in the basic approach. However, this energy is not uniformly distributed among the sensor nodes especially in the cases where the person moves only in the small part of the experiment area and where sensor nodes are deployed nonuniformly around the perimeter, e.g., the bookstore. To tackle this problem, we can consider the energy consumption of nodes in selecting the links within the circle or the ellipse. 


\section{CONCLUSION}

We introduced two effective energy efficient RTI approaches, ellipse-based and radius-based, for localization using RF sensor networks. In both energy efficient approaches, our aim was to save energy by reducing the number of links that we must measure to form an image of attenuation. In the ellipse-based approach, we only considered links in an ellipse around the velocity vector of the current position of the moving object and in the radius-based approach, we used links in a circle around the current position of the moving object. In addition, we proposed an algorithm to tune the radius of circle adaptively over time. We performed extensive evaluations using real experimental data from three different settings.

Our experimental results showed that our energy efficient approaches can save $50 \%$ to $80 \%$ of energy without seriously degrading localization accuracy. Interestingly, our radius-based approach even increased the accuracy of localization in comparison to the basic RTI approach.

\section{REFERENCES}

[1] N. Patwari and J. Wilson, "Rf sensor networks for device-free localization and tracking," Proceedings of the IEEE, vol. 98, no. 11, p 19611973, 2010.

[2] N. Patwari and P. Agrawal, "Effects of correlated shadowing: Connectivity, localization, and RF tomography," in IEEE/ACM Int'l Conf. on Information Processing in Sensor Networks (IPSN'08), April 2008, pp. 82-93.

[3] J. Wilson and N. Patwari, "Radio tomographic imaging with wireless networks," IEEE Trans. Mobile Computing, vol. 9, no. 5, pp. 621-632, May 2010, appeared online 8 January 2010.

[4] R. K. Martin, C. Anderson, R. W. Thomas, and A. S. King, "Modelling and analysis of radio tomography," in 2011 4th IEEE International Workshop on Computational Advances in Multi-Sensor Adaptive Processing (CAMSAP), Dec. 2011.

[5] O. Kaltiokallio and M. Bocca, "Real-time intrusion detection and tracking in indoor environment through distributed rssi processing," in IEEE 17th Intl. Conf. Embedded and Real-Time Computing Systems and Applications (RTCSA), vol. 1, Aug. 2011, pp. $61-70$.

[6] M. B. O. Kaltiokallio and N. Patwari, "Enhancing the accuracy of radio tomographic imaging using channel diversity," in IEEE Intl. Conf. on Mobile Ad hoc and Sensor Systems, 2012.

[7] J. Wilson and N. Patwari, "See through walls: motion tracking using variance-based radio tomography networks," IEEE Trans. Mobile Computing, vol. 10, no. 5, pp. 612-621, May 2011.

[8] Y. Zhao and N. Patwari, "Noise reduction for variance-based devicefree localization and tracking," in 8th IEEE Conference on Sensor, Mesh and Ad Hoc Communications and Networks (SECON'11), June 2011.

[9] O. Kaltiokallio, M. Bocca, and N. Patwari, "Follow @ grandma: Longterm device-free localization for residential monitoring," in IEEE International Workshop on Practical Issues in Building Sensor Network Applications, 2012.

[10] D. Zhang, J. Ma, Q. Chen, and L. M. Ni, "An RF-based system for tracking transceiver-free objects," in IEEE PerCom'07, 2007, pp. 135144.

[11] X. Chen, A. Edelstein, Y. Li, M. Coates, M. Rabbat, and A. Men, "Sequential Monte Carlo for simultaneous passive device-free tracking and sensor localization using received signal strength measurements," in ACM/IEEE Information Processing in Sensor Networks (IPSN), April 2011.

[12] Y. Zheng and A. Men, "Through-wall tracking with radio tomography networks using foreground detection," in Proc. Wireless Communications and Networking Conference (WCNC 2012), April 2012, pp. 32783283 .
[13] M. Moussa and M. Youssef, "Smart services for smart environments: Device-free passive detection in real environments," in IEEE PerCom09, 2009, pp. 1-6.

[14] J. Wilson and N. Patwari, "A fade level skew-Laplace signal strength model for device-free localization with wireless networks," IEEE Trans. Mobile Computing.

[15] D. Zhang, J. Ma, Q. Chen, and L. M. Ni, "Dynamic clustering for tracking multiple transceiver-free objects," in IEEE PerCom'09, 2009, pp. $1-8$.

[16] C. Xu, B. Firner, R. S. Moore, Y. Zhang, W. Trappe, R. Howard, F. Zhang, and N. An, "Scpl: indoor device-free multi-subject counting and localization using radio signal strength," in Proceedings of the 12th International Conference on Information Processing in Sensor Networks, April 2013, pp. 79-90.

[17] M. Bocca, O. Kaltiokallio, N. Patwari, and S. Venkatasubramanian, "Multiple target tracking with RF sensor networks," IEEE Transactions on Mobile Computing, 2013, appeared online 25 July 2013.

[18] F. Adib and D. Katabi, "See through walls with Wi-Fi!" in $A C M$ SIGCOMM'13, Aug. 2013.

[19] D. Maas, J. Wilson, and N. Patwari, "Toward a rapidly deployable rti system for tactical operations," in 8th IEEE International Workshop on Practical Issues in Building Sensor Network Applications (SenseApp 2013), Sydney, Oct. 2013

[20] Q. Pu, S. Gupta, S. Gollakota, and S. Patel, "Whole-home gesture recognition using wireless signals," in The 19th Annual International Conference on Mobile Computing and Networking (Mobicom'13), Sept. 2013.

[21] N. Patwari, J. Wilson, S. A. P.R., S. K. Kasera, and D. Westenskow, "Monitoring breathing via signal strength in wireless networks," arXiv.org, Tech. Rep. arXiv:1109.3898v1 [cs.NI], Sept. 2011.

[22] B. Mager, N. Patwari, and M. Bocca, "Fall detection using RF sensor networks," in IEEE Personal, Indoor and Mobile Radio Communications Conference (PIMRC 2013), London, Sept. 2013.

[23] S. Sigg, M. Scholz, S. Shi, Y. Ji, and M. Beigl, "RF-sensing of activities from non-cooperative subjects in device-free recognition systems using ambient and local signals," IEEE Trans. Mobile Computing, 2013, appeared online 21 Feb. 2013

[24] O. Kaltiokallio and M. Bocca, "Real-time intrusion detection and tracking in indoor environment through distributed RSSI processing," in 2011 IEEE 17th Intl. Conf. Embedded and Real-Time Computing Systems and Applications (RTCSA), vol. 1, Aug. 2011, pp. $61-70$.

[25] Y. Mostofi, "Cooperative wireless-based obstacle/object mapping and see-through capabilities in robotic networks," IEEE Transactions on Mobile Computing, vol. 12, no. 5, pp. 817-829, 2013.

[26] A. Gonzalez-Ruiz and Y. Mostofi, "Cooperative robotic structure mapping using wireless measurements: A comparison of random and coordinated sampling patterns," IEEE Sensors Journal, vol. 13, no. 7, pp. 2571-2580, 2013.

[27] M. A. Kanso and M. G. Rabbat, "Compressed RF tomography for wireless sensor networks: Centralized and decentralized approaches," in 5th IEEE Intl. Conf. on Distributed Computing in Sensor Systems (DCOSS-09), Marina Del Rey, CA, June 2009.

[28] W. Heinzelman, A. Chandrakasan, and H. Balakrishnan, "Energyefficient communication protocol for wireless microsensor networks," in In Proccedings of the Hawaii Conference on System Sciences, January 2000.

[29] S. W. X. Wang, J. J. Ma and D. W. Bi, "Cluster-based dynamic energy management for collaborative target tracking in wireless sensor networks," Sensors, vol. 7, 2007.

[30] J. Fuemmeler and V. Veeravalli, "Smart sleeping policies for energy efficient tracking in sensor networks," IEEE Trans. Signal Processing, vol. 56, no. 5, 2008.

[31] J. Wilson. Spin: Tinyos code for rss collection. [Online]. Available: http://span.ece.utah.edu/spin

[32] Texas Instruments. A USB-enabled system-on-chip solution for 2.4 $\mathrm{GHz}$ IEEE 802.15.4 and ZigBee applications. [Online]. Available: http://www.ti.com/lit/ds/symlink/cc2531.pdf

[33] CrossBow. Telosb data sheet. [Online]. Available: http://www.willow.co.uk/TelosB Datasheet.pdf 